\title{
Crónica de las repercusiones del Estudio: "El derecho administrativo ante la jurisprudencia de la Corte Suprema: líneas y vacilaciones", Publicado en esta Revista, N²8 (2019)
}

El Estudio "El derecho administrativo ante la jurisprudencia de la Corte Suprema: Líneas y vacilaciones Veinte temas, diez años (2008-2018)", publicado en el No 28 (2019) de esta Revista, en un número especial dedicado exclusiva y únicamente a este trabajo, generó diversas repercusiones a nivel nacional, tanto en el mundo académico como en la prensa.

Esta es una crónica de esas repercusiones. Puede verse, igualmente, en este número, la continuación de ese Estudio, en que se exponen las líneas y vacilaciones ocurridas durante el año 2019, el que sigue el mismo método y formato que el anterior.

\section{Publicación del Estudio en la ReDAE}

El Estudio fue dirigido por el académico e investigador Alejandro Vergara Blanco con la colaboración de un equipo de ayudantes del Programa de Derecho Administrativo Económico UC.

Se dedicaron 184 páginas de la Redae a la publicación de esa investigación, en la que se estudió y analizó casi 900 sentencias judiciales de la Corte Suprema relativas a 20 temas relevantes para el Derecho Administrativo, durante diez años (2008-2018), distinguiendo en cada materia los hechos que dan origen al conflicto y su regulación; la razón jurídica de la decisión (ratio iuris decidendi) y el escenario jurisprudencial. Además, se expusieron, respecto de cada materia analizada, dos clases de gráficos que mostraban de manera expresa el comportamiento de la sala y el comportamiento de los ministros y los abogados integrantes de la Corte Suprema.

\section{Discusión pública del tema y anticipo del Estudio en la prensa}

Previo a la publicación del Estudio en la ReDAE, hubo varios artículos en la prensa escrita nacional relativos a la investigación de la jurisprudencia, en las que se fue mostrando la evidencia de las vacilaciones jurisprudenciales, así como también, la importancia de poner en conocimiento de la opinión pública la prolongada ocurrencia de estos hechos, por parte del máximo tribunal del país, la Corte Suprema.

a) Columna de opinión. La primera aparición en la prensa del tema fue la columna "Vacilaciones jurisprudenciales", publicada el 27 de septiembre de 2019, en 
la sección "Espacio Abierto" del diario La Tercera, en la que el director del Estudio expuso el fenómeno de las vacilaciones jurisprudenciales y el zigzagueo al momento de resolver por parte de quienes integran las salas de la Corte Suprema, proponiendo además "que el Pleno de la Corte Suprema analice este asunto y emita, por ejemplo, un auto acordado que regule y sancione los cambios bruscos de jurisprudencia, y que se sancione a los jueces y abogados integrantes que, cual veletas al viento, cambian sus votos una y otra vez. También se podría dictar una ley contra esta mala costumbre de algunos jueces, cuyas vacilaciones jurisprudenciales cabe considerar abusivas y antidemocráticas".

b) Primer reportaje. Luego, el 10 de diciembre de 2019, se publica en el diario La Tercera, en la sección "Temas de hoy" el reportaje titulado "El zigzag de la Corte Suprema", en el que se expusieron los zigzagueos de los integrantes de la Corte Suprema, ministros y abogados integrantes, en cuatro materias relevantes para el Derecho Administrativo: medioambiente (plazo para la invalidación de una resolución de calificación ambiental), minería (requisitos previos para la constitución de una servidumbre minera), administrativo (cómputo de plazo para recurrir a tribunales por un acto administrativo) y derechos de aguas (regularización de derechos de aguas). El reportaje fue acompañado por una entrevista al director de la investigación relativa a las vacilaciones jurisprudenciales, la cual se tituló "Hay una especie de anarquía jurisprudencial".

c) Editorial y columna. A raíz de la publicación anterior, el día 14 de diciembre el diario El Mercurio publicó la editorial titulada "Sobre las sentencias de la Corte Suprema", en la que se comentó el Estudio realizado señalando "La jurisprudencia requiere de consistencia para otorgar certeza a aquellos justiciables que llevan sus problemas ante el conocimiento del Poder Judicial". Continuando con el interés de la prensa en el Estudio, el día 13 de febrero de 2019 el diario El Mercurio publicó, en su cuerpo A, la columna "Jurisprudencia de precedentes obligatorios" del académico director del Estudio, en la que señaló, entre otras cosas: "...Existe una percepción generalizada de que los vaivenes de la jurisprudencia son negativos y los primeros pasos para superarlo debiesen darlos cada juez, cada sala y el Pleno de la Corte Suprema...".

\section{Presentación oficial del Estudio en la Universidad}

Esta investigación fue oficialmente presentada en el seminario titulado "Líneas y vacilaciones de la Corte Suprema en Derecho Administrativo (2008-2018)" realizado el día 27 de marzo del 2019, en la Facultad de Derecho de la Pontificia Universidad Católica de Chile.

a) Participación académica. La presentación se dividió en tres paneles, todos compuestos por destacados académicos y abogados. En el primer panel presentó el Estudio el profesor y rector de la Universidad Diego Portales, Carlos Peña y se abordaron temáticas generales tales como: el rol de la jurisprudencia y los precedentes, la motivación, método y resultados del Estudio. Luego en el segundo y tercer panel se abordó, por parte de destacados académicos, de forma más específica la actualidad jurisprudencial en diferentes temas relevantes del Derecho Administrativo: función pública, procedimiento administrativo, derecho administrativo sancionatorio, derecho minero, derecho ambiental y derecho de aguas. Participaron en este seminario los académicos y abogados Alejandro Romero, Alejandro Cárcamo, Carolina Helfmann, Rosa Gómez, María Paz Pulgar y Edesio Carrasco.

b) Reportaje. A raíz de esta importante presentación, El Mercurio Legal realizó un acabado reportaje, el cual se publicó el día 28 de marzo del 2019 sobre lo presen- 
tado en el seminario "Líneas y vacilaciones...", exponiendo de manera pormenorizada lo expuesto por los destacados relatores que acudieron a la cita. También se publicó la columna del académico y rector de la Universidad Diego Portales, Carlos Peña, titulada "El maltrato de las reglas", en la cual comentó la importancia de un estudio de estas características, y propuso, para evaluarlo desde un punto de vista general, el examen de tres cuestiones que se encuentran entrelazadas entre sí: i) el sentido o el valor de las reglas; ii) las funciones de la dogmática; y iii) el derecho como profesión. Relativa a la misma temática, el mismo autor publicó la columna "Las reglas en peligro" en el cuerpo A del diario El Mercurio, el día 28 de marzo de 2019.

\section{Presentación del Estudio en otras universidades y sedes}

La investigación fue presentada por su autor en múltiples instancias, generando una amplia discusión sobre la temática de las vacilaciones jurisprudenciales, tanto respecto de su metodología, así como de las materias abordadas, y también respecto de la utilidad de esta clase de investigaciones en el ejercicio de la abogacía.

a) Facultad de Derecho de la Universidad de Chile. El Estudio se enfrentó al debate académico, con la especializada discusión de los administrativistas de la Facultad de Derecho de la Universidad de Chile, quiénes en un seminario especialmente dedicado a comentar esta investigación, realizado el día 12 de marzo del 2019 debatieron largamente sobre los resultados del Estudio, la labor de la judicatura en el Derecho Administrativo, el rol del precedente en nuestra jurisprudencia y la metodología de recopilación de las casi 900 resoluciones judiciales analizadas y estudiadas.

b) Colegio de Abogados de Chile. Posteriormente, el Estudio fue presentado por su autor el día 2 de abril de 2019 en la sesión inaugural del ciclo de charlas "Los martes al Colegio", organizado por el Colegio de Abogados de Chile, explicando de manera detallada los aspectos más relevantes del Estudio y con ello, abriendo el debate a todos aquellos que ejercen la abogacía.

c) Facultad de Derecho de la Universidad de Concepción. Se realizó un seminario sobre el Estudio en la Facultad de Derecho de la Universidad de Concepción el día 5 de abril de 2019, actividad que fue organizada por el Departamento de Derecho Público de la mencionada casa de estudios, dirigido por el académico Fabián Huepe.

d) Colegio de Abogados de Curicó. También se presentó en la comuna de Curicó, el día 12 de abril de 2019, ante el Colegio de Abogados de la ciudad.

e) Academia Judicial. La investigación también fue presentada a los futuros jueces, en un seminario organizado por la Academia Judicial para la exposición de la misma, realizado el día 29 de abril del 2019. Este contó además con un conversatorio con el vocero de la Corte Suprema (2019), el ministro Lamberto Cisternas (respecto de cuya intervención volvemos al final de estas líneas).

f) Facultad de Ciencias Jurídicas y Sociales de la Universidad de Talca. Además, el Estudio fue comentado por el decano de la Facultad de Ciencias Jurídicas y Sociales de la Universidad de Talca, el académico Raúl Carnevali en un seminario realizado especialmente para la divulgación de la investigación el día 2 de mayo de 2019.

g) Facultad de Derecho de la Pontificia Universidad Católica de Valparaíso. Por otra parte, la investigación fue presentada el día 10 de mayo de 2019 en la clase inaugural del Diplomado en Derecho Administrativo de la Facultad de Derecho de la Pon- 
tificia Universidad Católica de Valparaíso, cuyo director es el académico y abogado de la misma casa de estudios Eduardo Cordero.

h) Círculo Legal de ICARE. Por último, el martes 25 de junio de 2019, el Círculo Legal del Instituto Chileno de Administración Racional de Empresas (ICARE), presidido por la abogada Nicole Nehme, realizó el encuentro "Corte Suprema: ¿Jurisprudencia de precedentes?", en la Sede ICARE, en el que se presentó el Estudio, dando paso luego a un panel de conversación moderado por la presidenta del Círculo Legal, Nicole Nehme e integrado por Luis Cordero, abogado y académico de la Universidad de Chile, y Juan Ignacio Piña, abogado y académico de la UC.

\section{El Estudio en la prensa, después de su publicación (y reacciones de la vocería de la Corte Suprema)}

a) Reportaje. Luego de ser publicado el Estudio en la ReDAE, el diario El Mercurio, en su cuerpo C y El Mercurio Legal, sitio web especializado en materias jurídicas y legales, perteneciente al mencionado diario, publicó el día 9 de marzo el reportaje titulado "Estudio revela disparidad de criterio de la Suprema al fallar casos en materia administrativa". En ambas publicaciones, se presentó de manera inédita y completa el Estudio, acompañado además de una entrevista a su autor. En ella, el académico detalló cómo había surgido la idea de realizar este Estudio, así como las conclusiones de este y las razones que según él explican el comportamiento de los ministros y abogados integrantes de la Corte Suprema.

b) Intercambio epistolar. Ante la publicación del Estudio, el vocero de la Corte Suprema durante el año 2019, Lamberto Cisternas Rocha declaró al diario El Mercurio, el día 10 de marzo del mencionado año: "Nosotros estamos frente al caso concreto y lo resolvemos". En respuesta a la reacción manifestada en la declaración anterior del vocero de la Corte Suprema, el director de la investigación envió una carta al Director del diario El Mercurio, la que se publicó el día 11 de marzo, en la que se defendió la metodología utilizada para la recopilación y análisis de sentencias, la difusión del Estudio y critica la falta de una opinión crítica a la vocería de la Corte Suprema sobre el fenómeno de las vacilaciones. A lo anterior, respondió el vocero de la Corte Suprema, mediante una carta al Director, publicada el día 12 de marzo de 2019 en el diario El Mercurio, respondiendo a la misiva anterior, señalando, entre otras cosas: "Las bases técnicas y el perfil general de cualquier estudio son necesarios para calibrar su sentido, propósito y consistencia, lo que permite conversar y debatir a su respecto. No basta con la sola afirmación del autor sobre las bondades y efectos del Estudio. Esto lo sabe y acepta todo académico e investigador". Responde nuevamente el autor del Estudio, con una carta al Director, publicada el día 13 de marzo de 2019 en el diario El Mercurio a las críticas deslizadas por el ministro Cisternas en la carta anterior, agradeciendo las explicaciones relativas a las declaraciones realizadas a la prensa el día 10 de marzo y poniendo el énfasis en el debate al que deben llevar esta clase de investigaciones, poniéndose a disposición del mismo.

c) Unas declaraciones que definen el pensamiento judicial. En virtud del revuelo causado por el Estudio, el vocero de la Corte Suprema, Lamberto Cisternas Rocha, durante el seminario organizado por la Academia Judicial para la exposición de la investigación, realizado el día 29 de abril del año 2019, señaló lo siguiente:

"10 Lo que hacemos los jueces es fallar según la Constitución y la ley. Existen materias que debiesen tener una definición legal más precisa, pues eso origina dudas. 
$2^{\circ}$ Respecto de la posición de cada juez: Nuestro sistema es el 'del caso concreto' y no 'del precedente'. Y de pronto el juez se encuentra con casos que no son exactamente iguales; y debemos aplicar los principios.

$3^{\circ}$ Existe un 'problema institucional': las variaciones en la integración de las salas. Hay entonces una variación institucional.

$4^{\circ}$ El tema de la independencia del juez es relevante: los jueces somos muy quisquillosos con la independencia. Es la ley de la relatividad lo que rige.

$5^{\circ}$ El zigzagueo 'no viste bien', y se deben 'tomar las medidas para evitar este zigzagueo'. Debemos poner los mecanismos para evitar que este fenómeno suceda. Algunos tenemos el empeño por fijarnos en los dictámenes anteriores, de tal manera de mantener las líneas anteriores.

Agradecemos mucho estos estudios. Pero insisto en la independencia de cada juez y en los problemas institucionales (variaciones en la integración de las salas)."

\section{Comentarios finales}

La investigación objeto de esta crónica "El derecho administrativo ante la jurisprudencia de la Corte Suprema: Líneas y vacilaciones" generó amplias y diversas repercusiones a nivel nacional. Fue un Estudio innovador en cuanto a la materia, a la cantidad de sentencias y el período de tiempo analizados. De gran vanguardia además fue la inclusión de los gráficos que mostraban expresamente las tendencias de votación tanto de la Sala, como de los ministros y abogados integrantes de la Corte Suprema.

Muestra de lo anterior es que tanto académicos como litigantes mostraron opiniones de agradecimiento ante el exhaustivo trabajo realizado, enfatizando además en la relevancia de las resoluciones en nuestro sistema judicial, así como en el valor de la certeza y de un trato ecuánime al acudir a los tribunales de justicia. Además, los comentarios al Estudio fueron de enorme valor, al abrir el debate en temáticas como la independencia de los jueces, el rol del precedente en las resoluciones judiciales y el rol unificador del máximo tribunal del país, así como la eficiencia de los mecanismos legales existentes para llevar a cabo la unificación de la jurisprudencia.

La discusión recién ha comenzado, pero gracias a este Estudio, han quedado palpablemente expuestas varias debilidades de nuestro sistema judicial, fragilidades cuya relevancia se ha ido acrecentando mediante los comentarios y el debate que este trabajo generó.

Por último, el desafío actual supone hacerse cargo de la realidad evidenciada con este Estudio y que el debate procure entregar soluciones que ayuden a enfrentar, de manera transparente y democrática, el fenómeno de las vacilaciones jurisprudenciales.

\section{Carolina Carcaman Rodríguez}

Coordinadora Académica

Programa de Derecho Administrativo Económico

PDAE UC 